\title{
A computational approach to dendritic spine motility with calcium signaling by the immersed boundary method with advection-electrodiffusion Pilhwa Lee* and Charles S Peskin
}

Address: Courant Institute of Mathematical Sciences, New York University, New York, NY, USA

Email: Pilhwa Lee* - leep@cims.nyu.edu

* Corresponding author

from Sixteenth Annual Computational Neuroscience Meeting: CNS*2007

Toronto, Canada. 7-12 July 2007

Published: 6 July 2007

BMC Neuroscience 2007, 8(Supp| 2):P95 doi:I0.I I86/I47|-2202-8-S2-P95

(C) 2007 Lee and Peskin; licensee BioMed Central Ltd.

Dendritic spines are small protrusions from the dendritic branches of neurons. Influenced by internal and external signals and forces, even adult spines are not static but dynamically move. In this paper, we consider actomyosinbased spine motility with calcium signaling. The simulation begins with influx of calcium ions through glutamate receptors. Calcium Induced Calcium Release (CICR) with $\mathrm{IP}_{3}$ (inositol-1,4,5-trisphosphate) dynamics is also considered. The sensitivity of elasticity of actomyosin network is assumed to follow a Hill-type function of $\mathrm{Ca}^{2+}$ concentration. Several combinations in size of spine head and neck, physiology of Endoplasmic Reticulum (ER), and distribution of receptor/channels/exchangers are considered. Different functions of a spine as absorber, pumper and/or diffuser are observed. The computational framework used for these studies is the immersed boundary method with advection-electrodiffusion. 